\title{
Phase Operator and Condensed Systems
}

\author{
F. Rocca and M. Sirugue
}

Laboratoire de Physique Théorique, Université de Nice, Nice, France ${ }^{\star \star}$

Received March 30, 1973

\begin{abstract}
We prove the non existence of a self adjoint phase operator for systems with a finite number of degrees of freedom and for systems with an infinite number of degrees of freedom but enclosed in a finite box. We explicitely construct an example of a phase operator for an infinitely extended Bose system with condensation and give an application of this construction to a simple model.
\end{abstract}

\section{Introduction}

At the very early stage of quantum theory it has been tempting to apply the quantization rules to canonical variables other than the $p$ 's and $q$ 's. More precisely let us consider a one degree of freedom system; one can define the following classical new canonical variables:

$$
\begin{aligned}
& n=\frac{1}{2}\left(\varrho p^{2}+\varrho^{-1} q^{2}\right), \quad \varrho>0 \\
& \phi=\operatorname{Arctg}\left(\varrho \frac{p}{q}\right)
\end{aligned}
$$

whose classical Poisson bracket is:

$$
\{n, \phi\}=1 \text {. }
$$

So it was assumed [1] the existence of two self adjoint operators $N, \Phi$ such that

$$
[N, \Phi]=i \text {. }
$$

Such a commutation rule would lead to the well known incertainty relation

$$
\Delta N \cdot \Delta \Phi \geqq \frac{1}{2} .
$$

A lot of papers have been devoted to stress the difficulties (even contradictions) of the existence of $N$ and $\Phi$ with the assumed properties within the ordinary quantum mechanics (see for instance [2] for references; see also [3] for a solution of the problem of quantization).

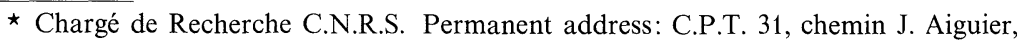
F-13009 Marseille, France.

$\star \star$ Equipe de Recherche Associée au C.N.R.S. 
In order to avoid the problems linked to the definition of an angular variable (defined up to $2 \pi$ ) Carruthers and Nieto introduced hermitian operators $C$ and $S$ which are the quantum analogues of $\cos \phi$ and $\sin \phi$ in the classical theory (see also [4] and enclosed references). Unfortunately those operators do not commute at least for systems with a finite number of degrees of freedom [5] and even for systems with an infinite number of degrees of freedom within the Fock representation [6]. Indeed in these situations the commutator is essentially the projection over the vacuum state of the system.

The non commutativity of $C$ and $S$ precludes the possibility of finding an unitary operator $U=C+i S$ and consequently a phase operator $\Phi$ satisfying (1.3) and (1.4).

Despite these drawbacks it is intuitively clear that the definition of such objects is relevant to some very interesting systems as superfluids and superconductors [7]. An approach of the problems linked to such systems using $C$ and $S$ has been done by some authors $[2,6,8]$.

Our goal in this paper is twofold: firstly to state the problem of the existence of an unitary operator $U=\exp (i \Phi)$ in its full generality, more precisely within an arbitrary representation of the canonical commutation relations. Secondly to give an explicit example of the existence of a phase operator within a representation which is physically interesting.

\section{The Problem of Existence of an Unitary Phase Operator}

We shall need a generalization of the notion of number operator for a representation of the Canonical Commutation Relations (C.C.R.) as it is developed for instance in [9].

Let $\mathscr{H}$ be the one particle space (its dimensionality is the number of degrees of freedom of the system) and denotes by $\langle\cdot\rangle$ its scalar product. A Weyl system over $\mathscr{H}$ is a correspondance $W$ from $\mathscr{H}$ to the unitary group of some complex Hilbert space $K$ such that.

i) $W(\psi) W\left(\psi^{\prime}\right)=\exp \left(\frac{i}{2} \operatorname{Im}\left\langle\psi \psi^{\prime}\right\rangle\right) W\left(\psi+\psi^{\prime}\right)$.

ii) $\lambda \in \mathbb{R} \rightarrow W(\lambda \psi)$ is weakly continuous at $\lambda=0$.

Creation operator $a^{*}(\psi)$ is defined as the closure of:

$$
\frac{1}{\sqrt{2}}(R(\psi)-i R(i \psi))
$$

where $R(\psi)$ is the infinitesimal generator of the group $t \rightarrow W(t \psi)$. 
Definition (2.2) (Chaiken). Let $W$ be a Weyl system over $K$. A self adjoint operator $N$ is a number operator for $W$ iff:

$$
\exp (i t N) W(\psi) \exp (-i t N)=W\left(e^{i t} \psi\right)
$$

$\forall \psi \in \mathscr{H}, \forall t \in \mathbb{R}$.

It is shown in [9] that one can normalize the spectrum of $N$ to be a subset of the integers $\mathbb{Z}$. We shall assume in the following that normalization.

The following theorem is an obvious consequence of the results in [9].

Theorem (2.3). Let $W$ be a Weyl system with at least one number operator; there are three possible disjoint cases:

i) All the different number operators are bounded from below; this occurs if and only if $W$ is a Fock representation (or a direct sum of a finite number of copies of the Fock representation).

ii) There exists at least one number operator whose spectrum is bounded from below but there exists at least another one whose spectrum is the whole set of integers $\mathbb{Z}$; this occurs if and only if $W$ is a direct sum of an infinite number of copies of the Fock representation.

iii) There is no number operator whose spectrum is bounded from below; this is equivalent to say that the representation is not quasi-equivalent to the Fock representation or equivalently that some subrepresentation of $W$ is disjoint to the Fock representation.

Let us make some remarks about this theorem.

i) The Definition (2.2) allows different numbers of particles when the representation is not irreducible. In that way (2.2) is a generalization of the usual definition of the number operator.

ii) The third case never appears when $\mathscr{H}$ is finite dimensional and this is due to von Neumann uniqueness theorem about representations of C.C.R.

iii) The number operator defined in (2.2) is not in general in the algebra generated by the $W(\psi)$ 's.

Let us define now that we shall call a phase operator.

Definition (2.4). A Weyl system $W$ has a phase operator with respect to a given number of particles $N$ if and only if there exists an unitary operator $U$ such that:

$$
\exp (i \alpha N) U \exp (-i \alpha N)=e^{-i \alpha} U
$$

Given such a $U$ there exists always a self adjoint operator $\Phi$ such that $U=\exp (i \Phi) ; \Phi$ and $N$ satisfy (1.3). Next result gives the general structure of systems with a phase operator. 
Theorem (2.5). Given on a Hilbert space $K$ a strongly continuous group of unitaries $\alpha \rightarrow \exp (i \alpha N)$ and an unitary operator $U$ such that

$$
\begin{aligned}
\exp (i \alpha N) U \exp (-i \alpha N) & =\exp (-i \alpha) U \\
\exp (2 i \pi N) & =1
\end{aligned}
$$

then there exists two Hilbert spaces $H_{1}\left(=l_{2}(\mathbb{Z})\right)$ and $H_{2}$ such that up to an unitary equivalence

moreover:

$$
\begin{aligned}
K & =H_{1} \otimes H_{2}, \\
\exp (i \alpha N) & =\exp \left(i \alpha N_{1}\right) \otimes 1, \\
U & =U_{1} \otimes 1,
\end{aligned}
$$

$$
\begin{aligned}
\left(\exp \left(i \alpha N_{1}\right) f\right)(n) & =e^{i \alpha n} f(n), \quad f \in l_{2}(\mathbb{Z}), \\
\left(U_{1} f\right)(n) & =f(n+1) .
\end{aligned}
$$

Proof. Let us define:

$$
V(\alpha, n)=e^{\frac{i \alpha n}{2}} e^{i \alpha N} U^{n}, \quad \alpha \in \mathbb{R}, n \in \mathbb{Z} .
$$

It is an unitary operator and the $V$ 's satisfy:

moreover:

$$
V(\alpha, n) V\left(\alpha^{\prime}, n^{\prime}\right)=\exp \left(\frac{i}{2}\left(\alpha^{\prime} n-\alpha n^{\prime}\right)\right) V\left(\alpha+\alpha^{\prime}, n+n^{\prime}\right)
$$

$$
V(\alpha+4 \pi, n)=V(\alpha, n) .
$$

Hence the $V$ 's are a generalized Weyl system over the Abelian group $G=T \times \mathbb{Z}$ ( $T$ is the group of complex numbers of modulus one) and the function from $G \times G \rightarrow T$

$$
(\alpha, n)\left(\alpha^{\prime} n^{\prime}\right) \rightarrow \exp \left(\frac{i}{2}\left(\alpha^{\prime} n-\alpha n^{\prime}\right)\right), \quad \alpha, \alpha^{\prime} \in\left[0,4 \pi\left[, n, n^{\prime} \in \mathbb{Z}\right.\right.
$$

defines an isomorphism of $G$ onto its dual group $\hat{G}$. Consequently Theorem (2.5) is nothing but the generalization of Von Neumann's uniqueness theorem $[10,11]$.

Corollary (2.11). If there is a phase operator, the spectrum of $N$ is $\mathbb{Z}$; each eigensubspace of $N$ has the same degeneracy. Consequently there exists no phase operator in the first case of Theorem (2.3), i.e. in the Fock representation.

As we said in the introduction, this result is well known and is connected with the fact that the polar part $V$ of the polar decomposition of the annihilation operator despite the fact that it satisfies (2.4) is not unitary (see e.g. [3]). 
Notice that the previous theorem shows that in general $U$ is not uniquely defined by (2.4). Namely in Theorem (2.5)

$$
U^{\prime}=U_{1} \otimes U_{2}
$$

where $U_{2}$ is an arbitrary unitary operator on $\mathrm{H}_{2}$, would satisfy (2.4).

We have excluded the first case of Theorem (2.3). In the second case it is in general possible to built an unitary operator $U$ which satisfies (2.2) since there exists at least a number operator $N$ whose spectrum is $\mathbb{Z}$. Let us give an explicit example.

Let $\mathscr{H}=\mathbb{C}$ (one degree of freedom) and $a_{F}^{ \pm}$the ordinary creation and annihilation operators of the usual unique Fock representation on $H_{F}$ (with number operator $N_{F}$ ). Let:

$$
\begin{gathered}
K=H_{F} \otimes H_{F}, \\
a^{ \pm}=a_{F}^{ \pm} \otimes 1 \quad\left(W(\psi)=W_{F}(\psi) \otimes 1\right), \\
N=N_{F} \otimes 1-1 \otimes N_{F} \quad\left(\exp (i \alpha N)=\exp \left(i \alpha N_{F}\right) \otimes \exp \left(-i \alpha N_{F}\right)\right) .
\end{gathered}
$$

Moreover if $\left\{\phi_{n}\right\}_{n \geq 0}$ is the orthonormal basis of eigenstates of $N_{F}$, a complete basis of $H_{F} \otimes H_{F}$ is given by the $\phi_{n} \otimes \phi_{m}$. Define now the operator $U$ on that basis as:

$$
\begin{array}{lll}
U \phi_{n} \otimes \phi_{m}=\phi_{n-1} \otimes \phi_{m} & \text { if } & n>m \\
U \phi_{n} \otimes \phi_{m}=\phi_{n} \otimes \phi_{m+1} & \text { if } & n \leqq m .
\end{array}
$$

It is an easy computation to show that (2.4) is satisfied.

This construction is not purely artificial since the previous representation occurs for instance to describe the equilibrium state at temperature $T \neq 0$ of an harmonic oscillator.

The mean value of any function $A$ of $p$ and $q$ is given by:

$$
\langle A\rangle=\operatorname{Tr}\left(\varrho_{\beta} A\right), \varrho_{\beta}=\frac{e^{-\beta H}}{\operatorname{Tr}\left(e^{-\beta H}\right)}, \quad H=\frac{1}{2}\left(p^{2}+q^{2}\right)
$$

and $\beta=(k T)^{-1}$; let $\exp \left(-\beta \varepsilon_{n}\right)$ be the eigenvalues of $\varrho_{\beta}$. As it is well known e.g. [12] the mean value (2.16) appears as the expectation value of $A$ in the vector of $H_{F} \otimes H_{F}, \Omega_{\beta}=\sum_{n} \exp \left(-\frac{\beta \varepsilon_{n}}{2}\right) \phi_{n} \otimes \phi_{n}$. The definition of $N$ we have chosen is the one which annihilates $\Omega_{\beta}$.

The $U$ we have defined in (2.15) has a poor physical meaning and according to a previous remark one could expect that another $U$ can 
be defined which would satisfy (2.4) and be physically relevant. Nevertheless we have the following result:

Proposition (2.17). Let $W$ be a Weyl system which is a direct sum of an infinite number of copies of the Fock representation. Then for any number operator whose spectrum is $\mathbb{Z}$ the unitary operators $U$ which satisfy (2.4) do not belong to the weak closure of the algebra generated by the $W(\psi)$ 's.

Proof. By definition any representation on $K$ of the C.C.R. quasi equivalent to the Fock representation is of the form (up to unitary equivalence)

$$
\begin{gathered}
K=H_{F} \otimes H_{2}, \\
W(\psi)=W_{F}(\psi) \otimes 1,
\end{gathered}
$$

hence any number operator on $K$ is of the form:

$$
N=N_{F} \otimes 1+1 \otimes A
$$

where $A$ is an operator on $\mathrm{H}_{2}$.

Consequently any operator $U$ satisfying (2.4) and belonging to the algebra generated by the $W(\psi)$ 's is of the form

$$
U=U_{1} \otimes 1
$$

where $U_{1}$ is an unitary operator on Fock space which moreover satisfies (2.4) with respect to $N_{F}$. Hence a contradiction with Corollary (2.11).

So despite the fact that we have solved the algebraic problem in that case the $U$ operators we have defined have no direct physical meaning and are of little use for the program we develop in the end of the first section. For these physical applications it seems necessary to impose at least that $U$ belongs to the weak closure of the algebra generated by the $W(\psi)$ 's in order to have a phase which is observable; more precisely:

Definition (2.22). A Weyl system has an observable phase operator with respect to a number operator $N$ if there exists an unitary operator $U$ such that

i) $U$ satisfies (2.4)

ii) $U \in(W(\psi) ; \psi \in \mathscr{H})^{\prime \prime}$ the weak closure of the algebra generated by the $W(\psi)$ 's.

Next result is an immediate consequence of the previous results:

Corollary (2.23). Let $W$ be a Weyl system with a number operator. There exists an observable phase operator only if the corresponding representation is not quasi-equivalent to the Fock representation. As a special consequence there exists no observable phase operator for systems with a finite number of degrees of freedom. 


\section{Examples of Weyl Systems with Observable Phase Operator}

The previous results suggest to look for Bose systems with an infinite number of degrees of freedom and moreover, according to the physical program of the introduction, to consider systems with some condensation property. Let us describe the model:

$\mathscr{H}=\mathscr{D}$ the space of complex valued and infinitely differentiable functions with compact support on $\mathbb{R}^{3}$ with the ordinary scalar product.

Let $\varrho$ be the following operator: $\widehat{\varrho} \psi(k)=\varrho(k) \hat{\psi}(k)$ where $\hat{\psi}$ is the Fourier transform of $\psi .0<\varrho(k)<1$ is a continuous function of $k$.

$K$ the representation space is

$$
K=H_{F} \otimes H_{F} \otimes M
$$

where $H_{F}$ is the Fock space and $M$ is the space of square integrable functions on the circle with respect to the measure $\frac{d \theta}{2 \pi}$.

Let $C$ and $S$ be the following operators from $M$ to $M$

$$
\begin{aligned}
& (C f)(\theta)=\cos \theta f(\theta), \quad f \in M, \quad \theta \in[0,2 \pi[, \\
& (S f)(\theta)=\sin \theta f(\theta)
\end{aligned}
$$

for $W(\psi)$ we define the following operator:

$$
\begin{aligned}
W_{r}(\psi)= & W_{F}\left((1+\varrho)^{1 / 2} \psi\right) \otimes W_{F}\left(\varrho^{1 / 2} \bar{\psi}\right) \\
& \otimes \exp \left(\operatorname{ir}\left(\hat{\psi}_{1}(0) C+\hat{\psi}_{2}(0) S\right)\right)
\end{aligned}
$$

where $W_{F}$ denotes the Fock representation and $\psi_{1}$ and $\psi_{2}$ are respectively the real and imaginary part of $\psi ; r$ is a non negative constant.

The cyclic vector of this representation is:

$$
\Omega=\Omega_{F} \otimes \Omega_{F} \otimes \mathbb{1}
$$

where $\Omega_{F}$ is the Fock vacuum and $\mathbb{1} \in M$ is the function identically one on the circle.

One can easily calculate the expectation values of products of creation and annihilation operators with respect to $\Omega$. As a special case we compute the kernels $\left\langle a_{k}\right\rangle,\left\langle a_{k}^{*} a_{k^{\prime}}\right\rangle$ which correspond respectively to the one and two points functions.

$$
\begin{gathered}
\left\langle a_{k}\right\rangle=0 \\
\left\langle a_{k}^{*} a_{k^{\prime}}\right\rangle=\varrho(k) \delta\left(k-k^{\prime}\right)+\frac{r^{2}}{2} \delta(k) \delta\left(k^{\prime}\right) .
\end{gathered}
$$


One easily shows that the number operator can be chosen as follows:

$$
N=N_{F} \otimes 1 \otimes 1-1 \otimes N_{F} \otimes 1+1 \otimes 1 \otimes\left(i \frac{d}{d \theta}\right)
$$

which has as spectrum the whole $\mathbb{Z}$ and annihilates the vector $\Omega$.

Now we define an observable phase operator through the unitary operator

$$
U=1 \otimes 1 \otimes(C+i S) .
$$

Unitarity and (2.4) are obvious. We are still left to prove that $U \in\{W(\psi)\}^{\prime \prime}$. To do this we remark first that the vector $\Omega$ is cyclic and separating with respect to the algebra $\mathscr{R}$ generated by the $W(\psi)$ 's (see e.g. [13]). Hence the commutant $\mathscr{R}^{\prime}$ is exactly generated by the following Weyl system (see $[13,14])$ :

$$
\begin{aligned}
W_{r}^{\prime}(\psi)= & W_{F}\left(\varrho^{1 / 2} \bar{\psi}\right) \otimes W_{F}\left((1+\varrho)^{1 / 2} \psi\right) \\
& \otimes \exp \left(-i r\left(\hat{\psi}_{1}(0) C+\hat{\psi}_{2}(0) S\right)\right) .
\end{aligned}
$$

But it is obvious that $U$ commutes with the $W_{r}^{\prime}(\psi)$ 's. Hence $U \in \mathscr{R}^{\prime \prime}$.

Such representations occur in the description of the ideal Bose gas below the critical temperature in the thermodynamic limit. The actual state of the ideal Bose gas below the critical temperature is given by

$$
\begin{aligned}
& \int_{0}^{\infty} \operatorname{dr}\left(r / \varrho_{0}\right) e^{-r^{2} / 2 \varrho_{0}}\left(\Omega \mid W_{r}(\psi) \Omega\right) \\
& =\exp \left(-\frac{1}{4} \int(1+2 \varrho(p))|\hat{\psi}(p)|^{2} d p-\frac{1}{2} \varrho_{0}|\hat{\psi}(0)|^{2}\right) .
\end{aligned}
$$

The density of the system is $\varrho=\varrho_{0}+\int d p \varrho(p)$ (see e.g. [13, 15]).

The Hilbert space corresponding to this state is just the direct integral of Hilbert spaces (3.1) with respect to the measure:

$$
d \mu(r)=r / \varrho_{0} e^{-r^{2} / 2 \varrho_{0}} d r
$$

and within that space it is easy to built an observable phase operator by summation of operators (3.8).

For the case $T=0$ the situation is a little bit different. One has to consider the following definitions:

$$
\begin{gathered}
K=H_{F} \otimes M, \\
W(\psi)=W_{F}(\psi) \otimes \exp \left(i r\left(\hat{\psi}_{1}(0) C+\hat{\psi}_{2}(0) S\right)\right), \\
\Omega=\Omega_{F} \otimes 1, \\
N=N_{F} \otimes 1+1 \otimes\left(i \frac{d}{d \theta}\right) .
\end{gathered}
$$

(See for instance [16].) As previously it is possible to write a phase operator

$$
U=1 \otimes(C+i S) .
$$


Moreover $U$ is observable if one remarks that ([13] and [16]):

$$
U=\varrho_{0}^{-1 / 2} \lim _{V \rightarrow \infty} a^{*}\left(\psi_{V}\right)
$$

where $\psi_{V}$ is the characteristic function of the volume $V \in \mathbb{R}^{3}$.

\section{Physical Interpretation}

In this section we want to show that the results we have got can be expected on physical considerations and also give a model of possible use of operators $U$.

The representations of the C.C.R. where we have constructed an observable phase operator correspond to systems in the thermodynamical limit. This is not surprising since from a known theorem (see [17]) systems enclosed within a finite box are associated with representations quasi equivalent to the Fock representation, where by Proposition (2.17) there is no observable phase operator. On the other hand one would expect the existence of an observable phase operator only for those systems which have a phase transition of some kind and this is evidently not the case for finite systems.

Let us remark there is a duality between our results and the theorem of Dell'Antonio, Doplicher, Ruelle [18] where the number operator appears as an observable only in the representations where it is impossible to construct an observable phase operator. (The number operator we have considered to construct $U$ is never within the algebra of observables.)

Another remark is that in the examples we have displayed the observable phase operators are actually in the center of the algebra $\mathscr{R}$. On physical grounds it seems likely to conjecture this is a general situation.

Finally let us describe a crude model to show the interest of the definition of observable phase operator.

Let us consider two infinitely extended Bose systems at $T=0, \Sigma_{1}$ and $\Sigma_{2}$. Both are described by representations of the type (3.12) to (3.16). As representation space for coupled system we have:

$$
K=K_{1} \otimes K_{2}=H_{F}^{1} \otimes M^{1} \otimes H_{F}^{2} \otimes M^{2} .
$$

There are two different observable phase operators $U_{i}$

$$
\begin{aligned}
& U_{1}=1 \otimes(C+i S) \otimes 1 \otimes 1 \\
& U_{2}=1 \otimes 1 \otimes 1 \otimes(C+i S) .
\end{aligned}
$$


We take the hamiltonian of the complete system:

where:

$$
H=H_{0}+\frac{1}{2} g\left(U_{1} U_{2}^{*}+U_{2} U_{1}^{*}\right)
$$

$$
H_{0}=\mu_{1} N_{F}^{1} \otimes 1 \otimes 1 \otimes 1+\mu_{2} 1 \otimes 1 \otimes N_{F}^{2} \otimes 1, \quad \mu_{i}>0 .
$$

According to (3.17) that definition of the interaction corresponds physically to the exchange of particles between the condensed phases of each system.

Let us define the following operator

$$
\begin{aligned}
\Delta N= & \frac{1}{2}\left[N_{F}^{1} \otimes 1 \otimes 1 \otimes 1+1 \otimes\left(i \frac{d}{d \theta}\right) \otimes 1 \otimes 1\right. \\
& \left.-1 \otimes 1 \otimes N_{F}^{2} \otimes 1-1 \otimes 1 \otimes 1 \otimes\left(i \frac{d}{d \theta}\right)\right] .
\end{aligned}
$$

Then let us compute:

$$
\begin{aligned}
\Delta \dot{N} & =i[H, \Delta N] \\
& =\frac{1}{2} g i\left(U_{1} U_{2}^{*}-U_{2} U_{1}^{*}\right) .
\end{aligned}
$$

This operator will be interpreted as the flow $J$ of particles between the two systems; if we define $\Phi_{1}$ and $\Phi_{2}$ as the phase operators corresponding to $U_{1}$ and $U_{2}$ respectively then we get the following result using commutativity between $U_{1}$ and $U_{2}$ :

$$
J=g \sin \left(\Phi_{2}-\Phi_{1}\right) .
$$

This model simulates the interaction between two superfluid systems (or between two superconductors where the Cooper pairs are considered as Bose particles) and formula (4.5) is typical of the Josephson effect [19].

Acknowledgment. It is a pleasure for us to thank Prof. D. Testard and A. Verbeure for discussions.

\section{References}

1. Dirac, P.A. M.: Proc. Roy. Soc. London A 114, 243 (1927).

2. Carruthers, P., Nieto, M.M.: Rev. Mod. Phys. 40, 411 (1968)

3. Sirugue, M., Verbeure, A.: On the quantum analogue of a classical canonical transformation. Preprint Leuven University Belgium (1972)

4. Ifantis, E. K.: J. Math. Phys. 12, 1021 (1971)

5. Carruthers, P., Nieto, M. M.: Phys. Rev. Letters 14, 387 (1965)

6. Viano, M.: Thesis, Nice University (1973)

7. Feyman, R.P.: Lectures on physics, Vol. III. Reading, Mass.: Addison Wesley Publ. Co. 1965

8. Nieto, M.M.: Phys. Rev. 167, 416 (1968)

9. Chaiken, J. M.: Commun. math. Phys. 8, 164 (1968)

10. Mackey, G. W.: Duke Math. J. 16, 313 (1949) 
11. Slawny, J.: On the regular representation, Von Neumann uniqueness theorem and the $C^{*}$ algebra of canonical commutation and anticommutation relations. Preprint Tel Aviv University (1971)

12. Winnink, M.: Cargese lectures on physics, Vol. 4. New York: Gordon and Breach 1970

13. Araki,H., Woods, E.J.: J. Math. Phys. 4, 637 (1963)

14. Haag, R., Hugenholtz, N. M., Winnink, M.: Commun. math. Phys. 5, 215 (1967)

15. Wieringa, J.D.: Thesis, Gröningen University (1970)

16. Hugenholtz, N.M.: Fundamental problems in statistical mechanics, II. Amsterdam: North Holland Publ. Co. 1968

17. Ruelle,D.: Cargese lectures in theoretical physics. Gordon and Breach Science Publ. 1965

18. Dell'Antonio, G. F., Doplicher, S., Ruelle,D.: Commun. math. Phys. 2, 223 (1966)

19. Josephson, B. D. : Rev. Mod Phys. 36, 216 (1964)

\section{Sirugue}

C.P.T.

31, Chemin J. Aiguier 13274-Marseille Cedex 2

France
F. Rocca

Faculté des Sciences de Nice

Laboratoire de Physique Théorique

Parc Valrose

F-06 Nice, France 
